\title{
Creating a health equity and inclusion office in an academic pediatric medical center: priorities addressed and lessons learned
}

\author{
Valerie L. Ward ${ }^{1,2,3,4} \cdot$ Nicole W. Tennermann ${ }^{2} \cdot$ Geeranan Chuersanga ${ }^{2} \cdot$ Patrice Melvin 2,3,5 $^{\text {Maxine E. Milstein }}{ }^{6}$. \\ Jonathan A. Finkelstein ${ }^{4,7,8}$. Michele M. Garvin ${ }^{9} \cdot$ Laura J. Wood $^{10} \cdot$ Nina A. Rauscher ${ }^{11} \cdot$ Peter C. Laussen $^{4,11,12}$. \\ Alan M. Leichtner ${ }^{4,8,13,14}$. S. Jean Emans ${ }^{4,6,8,15} \cdot$ Kevin B. Churchwell ${ }^{4,16}$
}

Received: 1 April 2021 / Revised: 21 November 2021 / Accepted: 10 January 2022 / Published online: 1 March 2022

(c) The Author(s), under exclusive licence to Springer-Verlag GmbH Germany, part of Springer Nature 2022

\begin{abstract}
Background Over the last two decades, medical schools and academic health centers have acknowledged the persistence of health disparities in their patients and the lack of diversity in their faculty, leaders and extended workforce. We established an Office of Health Equity and Inclusion (OHEI) at our pediatric academic medical center after a thorough evaluation of prior diversity initiatives and review of faculty development data.

Objective To describe the lessons learned at a pediatric academic medical center in prioritizing and implementing health equity, diversity and inclusion (EDI) initiatives in creating the OHEI.

Materials and methods We reviewed internal administrative data and faculty development data, including data related to faculty who are underrepresented in medicine, to understand the role of our EDI initiatives in the strategic priorities addressed and lessons learned in the creation of the OHEI.

Results The intentional steps taken in our medical center's strategic approach in the creation of this office led to four important lessons to improve pediatric health equity: (1) board, senior executive and institutional prioritization of EDI initiatives; (2) multi-specialty and interprofessional collaboration; (3) academic approach to EDI programmatic development; and (4) intentionality with accountability in all EDI initiatives.

Conclusion The key lessons learned during the creation of an Office of Health Equity and Inclusion can provide guidance to other academic health centers committed to implementing institutional priorities that focus their EDI initiatives on the improvement of pediatric health equity.
\end{abstract}

Keywords Diversity $\cdot$ Health care disparities $\cdot$ Health equity $\cdot$ Inclusion $\cdot$ Pediatric $\cdot$ Underrepresented in medicine

\section{Introduction}

Over the last two decades, medical schools and academic health centers have acknowledged the persistence of health disparities in their patients and the lack of diversity in their faculty, leaders and extended workforce. With the devasting impact of health disparities illuminated by the 2020 confluence of the coronavirus disease 2019 (COVID-19) pandemic and the national reckoning for racial justice, the attainment of health equity and inclusion has taken on an even greater significance in the delivery of health care. Health equity has been defined as everyone having a fair and just opportunity

Valerie L. Ward

valerie.ward@childrens.harvard.edu

Extended author information available on the last page of the article to be as healthy as possible [1]. With that simple but broad definition of health equity, it is essential that our pediatric health care delivery inherently values all individuals and populations equally, recognizes and rectifies historical injustices, provides resources according to need, and aims to reduce and eliminate disparities in health and its determinants that adversely affect excluded or marginalized groups $[1,2]$. As a result, academic medical centers, hospitals, medical schools and clinical departments (including radiology, neurology, cardiology and others [3-6]) created pipeline and mentoring programs, sponsored educational efforts directed at selection committees to increase awareness of implicit bias in interviews and recruitment [7], and funded formal offices to address these gaps and to increase workforce diversity $[3,8]$. 
In this context, our pediatric academic medical center established an Office of Health Equity and Inclusion (OHEI) that is an enterprise-level office reporting directly to senior executive hospital leadership. In this paper, we present actionable and intentional steps taken as a part of our pediatric academic medical center's equity, diversity and inclusion (EDI) strategy to engage all sectors of its workforce and to implement institutional priorities and enterprise-wide goals focused on improving pediatric health equity.

In creating the OHEI, we learned four important lessons that are the focus of this paper. The successful creation of this office leveraged multi-specialty, interprofessional collaborations among pediatricians, pediatric subspecialists (including pediatric radiologists), nurses, interprofessional colleagues, researchers, administrators and senior hospital executive leaders. Given the potential for multiple layers of complexity and competing demands for resources and expertise, it was clear that the institutional EDI priorities and strategies needed to be intentional so as not to be easily overlooked by competing priorities [1].

\section{Materials and methods}

To delineate the priorities addressed and lessons learned as the OHEI was created and the scope of its work was expanded, we collected and reviewed data as described in the following subsections.

\section{Review of internal equity, diversity and inclusion administrative data}

To understand the work that had already been completed and to inform future opportunities for innovative EDI-related work, we reviewed these internal administrative data: annual reports of the Office of Faculty Development (unpublished documents), Diversity and Cultural Competency Council white paper 2011 (unpublished document) and faculty surveys administered by the Office of Faculty Development in 2017 and 2019 (unpublished data).

\section{Evaluation of educational equity, diversity and inclusion symposia survey data}

To evaluate the impact of educational programming in health equity and inclusion, we collected quantitative evaluation survey data from attendees at a continuing medical education symposium called the "Annual Boston Children's Hospital Pediatric Health Equity and Inclusion Symposia" annually from 2018 to 2020 . The symposium evaluation form included nine Likert scale questions with a scale from 1 (lowest) to 5 (highest) and an open-ended field for feedback and future suggested topics. The items were used to assess applicability and clarity of the material presented, speaker and venue attributes, whether learning objectives were met, and overall training and speaker satisfaction. To measure improvement in OHEI symposium evaluation results, we aggregated item-specific and overall Likert scores of 4 or 5 (on a Likert scale of 1 to 5) into a dichotomous positive response group. We assessed differences in positive response percentages across years using chi-square tests and trends using Cochran-Mantel-Haenszel tests.

\section{Assessment of the state of underrepresented-faculty diversity}

To assess the state of faculty diversity and the need for intentional career-development programs for faculty who are underrepresented in medicine, we evaluated our medical center's annual data on academic promotions. This evaluation included calculating the percentages of faculty at each academic rank who are underrepresented in medicine (Black/African American, Hispanic/Latinx and other groups).

\section{Assessment of impact of research grants on underrepresented-faculty retention and advancement}

To understand the impact of dedicated clinical and basic science research grants on the retention and academic advancement of faculty who are underrepresented in medicine, we reviewed the following data: grant awardees' demographic characteristics, academic rank at the time of funding, and subsequent promotions and retention, and whether faculty who are underrepresented in medicine obtained additional funding compared to non-underrepresented faculty. Using chi-square tests, we assessed differences in gender, retention, promotion and receipt of additional funding among Career Development Fellows according to their representation-inmedicine status (under-represented or not). A $P$-value less than 0.05 was considered statistically significant for all analyses. All analyses were performed using SAS version 9.4 (SAS Institute, Cary, NC).

\section{Results}

After reviewing data, institutional efforts to date, and programmatic initiatives, we identified four key lessons that were essential to our progress in advancing EDI outcomes over the last 20 years of EDI initiatives. 


\section{Lesson 1: prioritize health equity, diversity and inclusion initiatives as strategic goals endorsed by the board and senior executive leadership}

Boston Children's Hospital is the major pediatric teaching hospital of Harvard Medical School. Since 1968, Harvard Medical School has succeeded in recruiting a diverse student body, but the recruitment and retention of underrepresentedin-medicine faculty at both the Harvard Medical School quadrangle and the Harvard-affiliated teaching hospitals have lagged in comparison.

Over the last 20 years, the major Harvard Medical School teaching hospitals have created offices of faculty development, and a variety of diversity and health-equity programs and offices that are combined or collaborated closely with the faculty development efforts. In 2001, Boston Children's Hospital created the Office of Faculty Development, naming S. Jean Emans, MD, as director. From the beginning, the mission of the Office of Faculty Development has been to recruit and retain the best faculty, facilitate career advancement and satisfaction, and increase leadership opportunities, particularly for women and underrepresented-in-medicine faculty. The Office of Faculty Development has focused on fostering the careers of faculty at Boston Children's Hospital through career conferences, seminars, curriculum vitae review, 2-year career development fellowships - with some designated for underrepresented-in-medicine faculty or those with added responsibilities for child care or elder care, mentoring models, a family and career network, library resources, a newsletter, a dedicated website and collaborations with other offices [8].

Our medical center has a long history of and commitment to EDI initiatives that have been led by our Office of Faculty Development, Human Resources senior leaders, employee resource groups and specific leaders and individuals with EDI expertise and a commitment to this work. Boston Children's Hospital also has a history of having diversity committees. In 2006, senior leadership created a formal committee on diversity and inclusion called the Diversity and Cultural Competency Council. The Council led the EDI work under the guidance of the Office of Faculty Development and a faculty co-chair, Valerie L. Ward, MD, MPH, a pediatric radiologist, along with a Human Resources cochair. The work of this council has been governed by an executive steering committee with senior leadership representation. (In 2019, the Diversity and Cultural Competency Council revised its mission statement and was renamed the EDI Council.)

The Council faculty co-chair and the Office of Faculty Development represented Boston Children's Hospital at Harvard Medical School diversity and pipeline initiatives and national conferences such as that of the National Medical Association. The Council also undertook scholarly endeavors including tracking patient experience by race/ ethnicity, obtaining grant funding and publishing scientific studies that addressed health disparities. For example, the Council was awarded funding from the Blue Cross Blue Shield Foundation of Massachusetts to complete a "Pathways to Cultural Competency Grant" project to improve diverse patient navigation in the academic pediatric medical center. Council members within its health care disparities research working group collaborated with hospital administrative and executive leaders on two patient-centered studies that were funded by grants from the hospital's Program for Patient Safety and Quality. These studies resulted in publications focused on patient navigation and access $[9,10]$.

The accomplishments of the Council were recognized by Kevin B. Churchwell, MD, current Boston Children's Hospital president and chief executive officer, and Sandra L. Fenwick, MPH, retired Boston Children's Hospital chief executive officer. Yet, building on the successful experiences in establishing offices of equity, diversity and inclusion at two other pediatric academic medical centers, Dr. Churchwell knew having a dedicated office would be critical to strengthening the delivery of culturally effective pediatric health care at Boston Children's Hospital. In 2017, the Office of Health Equity and Inclusion was founded with Dr. Ward as its medical director. From 2017 to 2020, Boston Children's Hospital's EDI clinical, research and educational initiatives increased significantly, and in 2020 the senior hospital executive leaders recognized the need for Boston Children's Hospital to have its first chief equity and inclusion officer, and Dr. Ward was subsequently promoted to this senior executive enterprise-wide leadership position.

The creation of the Office of Health Equity and Inclusion and the elevation of its leadership underscored the commitment by senior hospital executive leaders that EDI initiatives must be thoughtfully structured and resourced. Prioritizing of EDI initiatives into every aspect of our medical center's strategic corporate goals was one expression of this intentionality. The prioritization of this work coincided with the June 2020 declaration by former Mayor Martin J. Walsh of the City of Boston, MA, that "racism is a public health crisis" in the setting of national discussions surrounding racial injustices and widening health care disparities that were amplified by the COVID-19 pandemic. The lesson learned here can be summarized as follows: effective prioritization of EDI initiatives must be a defined commitment and clear priority of the Board of Trustees, leadership at the level of the hospital president and chief executive officer, and key multidisciplinary stakeholders [1]. As such, our medical center's Board of Trustees and senior executive hospital leadership endorsed a 2020 Declaration on Equity, Diversity and Inclusivity. Figure 1 shows the six goals that comprise the Boston Children's commitments in its 2020 Declaration on Equity, Diversity and Inclusivity that are elevated 


\section{Boston Children's Hospital Declaration on Equity, Diversity and Inclusivity}

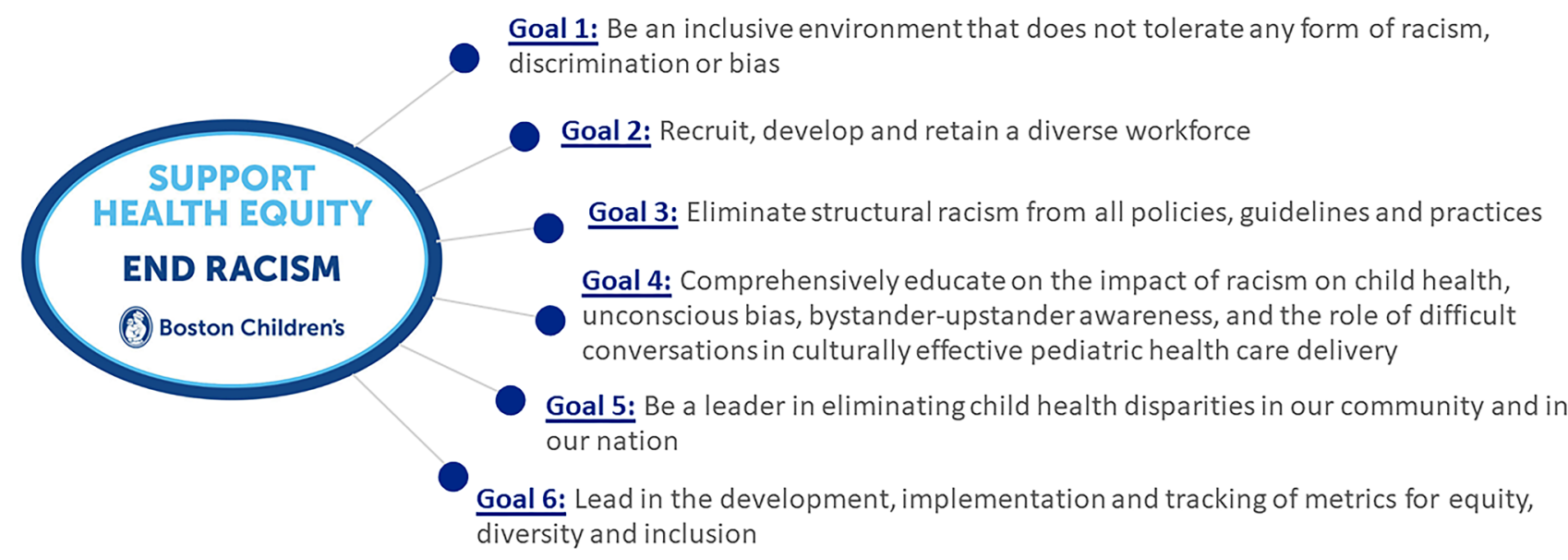

Fig. 1 Boston Children's Hospital 2020 Declaration on Equity, Diversity and Inclusivity goals

as strategic enterprise priorities, deeply embedded into its corporate goals and mission, and provide a clear present and future roadmap for all employees, staff, trainees, physicians, researchers, patients and families on how our academic medical center will work together to support pediatric health equity and commit to being an inclusive working and learning environment.

\section{Lesson 2: collaborate with multidisciplinary departments, programs, offices and experts}

\section{New and essential collaborations within Boston Children's Hospital}

A very important lesson that has contributed to our cumulative success has been intentional collaboration with key stakeholders and subject matter experts across our medical center. These collaborations provided a foundation for weaving EDI initiatives into almost every aspect of our health care delivery. Key collaborations included the Department of Health Affairs, Nursing and Patient Care Operations, Department of Education, Boston Children's Hospital Academy for Teaching and Educational Innovation and Scholarship, Office of the General Counsel, Office of Experience, and Program for Patient Safety and Quality. In addition to these new collaborations, the longstanding collaborations with the Office of Faculty Development and Human Resources on EDI initiatives continued to develop with the creation of the OHEI.

The collaboration with the Program for Patient Safety and Quality is focused on creating pediatric health equity-quality metrics to provide the accountability that is required to assess our delivery of culturally effective pediatric health care. This work aligns with goal 6 of the 2020 Boston
Children's Hospital Declaration on EDI, which outlines our commitment to developing, implementing and tracking health equity-quality metrics. Collecting and understanding these data are linked directly to our commitment to identify and mitigate pediatric health care disparities and to develop interventions to eliminate them. Additionally, the OHEI collaborated closely with the Program for Patient Safety and Quality, Office of Experience, and the Information Services Department to improve the collection of race, ethnicity and language data [11] from our patients. With a deeper understanding of our patients' demographic characteristics [11], the OHEI, Program for Patient Safety and Quality and other clinical and research collaborators can perform assessments of our health care interventions. In short, we recognized that an intentional collaboration between the experts in health equity and the experts in quality and safety was essential; without an effective measurement and data collection strategy, there would be no accountability for the effects of health care policies or programs [1].

\section{Harvard Medical School collaborations}

The OHEI and Office of Faculty Development collaborate closely with Harvard Medical School's Office for Diversity Inclusion and Community Partnership to support underrepresented-in-medicine faculty recruitment and develop networks and to support programs of interest to our underrepresented-in-medicine community. One of the goals of the collaboration is to promote efforts to link underrepresentedin-medicine students with Boston Children's Hospital faculty, advisers and underrepresented residents and fellows. The programs at Harvard Medical School work synergistically with Boston Children's Hospital's recruitment/retention efforts, including faculty fellowships and other funding 
for promising pilot research projects, special programs and networking events for trainees and faculty.

\section{National collaborations: Pediatric Health Equity Collaborative}

The Pediatric Health Equity Collaborative is an offshoot of Massachusetts General Hospital's Disparities Leadership Program and the Health Care Quality and Equity Action Forum. The Pediatric Health Equity Collaborative comprises faculty and staff from 12 institutions with offices of equity, diversity and inclusion [12]. With the creation of the OHEI in 2017, Boston Children's Hospital was able to join the Pediatric Health Equity Collaborative, and this collaborative focuses on establishing best practices in data collection in pediatric care settings. The OHEI's collaborations with this collaborative's member institutions include benchmarking of EDI initiatives and the exchange of best practices especially as they relate to race, ethnicity and language data collection and for health equity research studies and interventions [13]. This national collaborative of health-equity subject matter experts regularly shares important learnings and experiences to inform work within their own medical centers. At the 2018 conference of this collaborative, members presented Dr. Churchwell, Boston Children's Hospital's president and chief executive officer, with an award honoring his contributions as a "visionary leader and champion for pediatric health equity" for having created three offices of health equity, diversity and inclusion, with these offices comprising 3 of the 12 Pediatric Health Equity Collaborative's member institutions.

\section{Lesson 3: take an academic approach and create educational and research health equity, diversity and inclusion initiatives}

In goal 4 of the 2020 Boston Children's Hospital Declaration on EDI, we committed to being an inclusive hospital learning environment. We developed annual enterprise-wide symposia, visiting professorships in health equity and inclusion, grand rounds, educational workshops, and listening and discussion sessions.

Our review of the quantitative data from the evaluations of the continuing medical education annual Boston Children's Hospital Pediatric Health Equity and Inclusion Symposia (2018-2020) underscored the positive impact. In 2020, the third annual symposium was in honor of Women in Medicine and Science Month, and was a collaboration with the annual keynote speaker planned by the OHEI and Office of Faculty Development. In these annual symposia, we intentionally invited speakers who would add diversity and inclusivity.

The subjects of the symposia were:
- 2018, "What is Health Equity and How Can We Promote Equitable Pediatric Healthcare?"

- 2019, "Health Equity in Pediatric Subspecialty Care"

- 2020, "Women in Medicine and Science"

Evaluation of 3 years of symposia data (2018-2020) showed high scores with statistically significant increases in the positive ratings for speakers (90\% in 2018 to $99 \%$ in 2020 ) and for the program overall (94\% in 2018 to $100 \%$ in 2020). The percentage of symposium participants who responded positively ( 4 or 5 on a scale of 1 to 5 ) within and across the individual evaluation items (including material presented, speaker, session format and learning objectives) increased in the last 3 years. Overall, 97\% of participants in 2020 rated the symposium positively compared to $93 \%$ in $2018(P \leq 0.001)$. In 2020, positive item scores ranged from $87 \%$ to $100 \%$, with material applicability in the lower end and speaker's organization/preparation and overall rating receiving top ratings (symposium evaluation data not published).

We also created educational programming in health equity and inclusion in the form of a visiting professorship that was a collaboration with our hospital's Medical Staff Organization and Department of Health Affairs.

The OHEI sponsors recurring health-equity-focused grand rounds lectures, including Black History Month Grand Rounds (annually in February). Similar collaborations with other employee-led resource groups have enhanced the opportunities for new educational events, observances and lectures. For example, these have included the Boston Children's Hospital Rev. Dr. Martin Luther King Jr. Observance with a scholarly lecture in honor of Dr. King's legacy (annually in January), and educational and research programming for Lesbian, Gay, Bisexual, Transgender and Queer Health Awareness Month (annually in March).

In collaboration with the OHEI, the Boston Children's Hospital Academy of Teaching and Educational Innovation and Scholarship [14] and colleagues from the Department of Radiology, Division of Gastroenterology and the Simulation Program created an inclusive hospital workshop training for all faculty, staff, employees and trainees. This workshop is focused on unconscious biases and takes attendees from an introductory understanding to a more advanced examination of unconscious biases in the workplace. The program supports participants to consider effective responses when confronted with behaviors of bias, microaggression or macroaggression. Participants are equipped with safe and empowering strategies to go from being "bystanders" to "upstanders."

At Boston Children's Hospital, we also offered a supportive safe space, in a facilitated discussion setting, for all to share their thoughts and reflections during the 2020 national events of racial injustice and the remote work environment caused by the COVID-19 pandemic. During these listening 
and discussion sessions, attendees were encouraged to share their personal experiences and ideas about how we can promote an inclusive hospital environment and continue an ongoing conversation to enhance EDI for all.

In goal 5 of the 2020 Declaration on EDI, Boston Children's Hospital committed to being a leader in eliminating child health disparities in our community and in the nation. Aligning with this commitment, new opportunities for research collaborations and projects on health equity and health outcomes were developed in the OHEI. Also, multiple departments and individuals consulted the OHEI for subject matter expertise, pediatric health disparities research collaboration, and statistical consultation from a senior biostatistician who is a staff member in the OHEI.

\section{Medical Staff Organization pediatric health equity grant}

In 2019, in collaboration with the Medical Staff Organization and the Office of Faculty Development, the OHEI launched a pediatric health equity grant. This 1-year grant provides funding to faculty with projects focused on improving child health outcomes. The awardee also receives career and research mentorship from faculty in the OHEI and Office of Faculty Development.

\section{Pediatric health equity fellowship}

In 2020 at Boston Children's Hospital, we created and funded an innovative pediatric health equity fellowship program. The 2-year fellowship includes funding for a master's of public health degree at the Harvard T.H. Chan School of Public Health, and offers a dedicated health services research methodology curriculum within the Harvard-wide Pediatric Health Services Research Fellowship Program. The faculty awardee has opportunities to attend seminars and lectures in pediatric health equity at Harvard Medical School and Boston Children's Hospital and receives career and research mentorship from faculty in the OHEI and the Health Services Research Fellowship Program.

\section{Healthcare Disparities Research Working Group}

The Healthcare Disparities Research Working Group was convened at the request of a pediatric subspecialist who envisioned the need and interest for quarterly "works-inprogress" presentations by researchers across our medical center who are interested in improving health outcomes. The purposes of this group are to receive useful feedback on ongoing projects and to foster opportunities for research collaborations.

\section{Lesson 4: commit to intentionality and accountability for all equity, diversity and inclusion initiatives}

Boston Children's Hospital undertook intentional efforts to address the diversity of its workforce pipeline, including the development of innovative science, technology, engineering and math pipeline programs for college students and area high school students. The transition to remote work settings during the COVID-19 pandemic resulted in an opportunity to move these pipeline programs to the virtual work environment. Whether in-person or virtual, the purposes of these pipeline programs are to introduce students to clinical, administrative and research careers at Boston Children's Hospital and encourage them to pursue careers they might not have considered. Furthermore, the OHEI has purposefully expanded its collaboration with the Human Resources Department including sponsorship of underrepresented administrators and faculty for programs that focus on leadership development for diverse professionals.

For underrepresented-in-medicine faculty development programmatic planning, the OHEI and Office of Faculty Development performed a review of both the faculty development literature and medical education literature. This yielded important takeaways. For example, although diversity of clinicians in the workforce has been linked to better access and health outcomes for underrepresented populations $[15,16]$, program training directors might not select diversity as a top priority for their institutions [4]. At the same time, newly established programs or offices of health equity or diversity might focus on only one or two EDI initiatives that do not become integral to the strategic priorities of the institution. A study of diversity initiatives [17] found that the presence of an underrepresented-in-medicine faculty development program, per se, was not associated with higher levels of underrepresented faculty, recruitment or promotion; instead, programs with 5 or more years of experience and greater intensity with multiple components did have an impact, with greater increases in underrepresented faculty [18]. The lack of progress in the percentage of underrepresented faculty among academic faculties [2] and a new urgency to address structural racism have accelerated the pace and importance of reviewing programs with intentional multi-faceted efforts $[19,20]$.

At our medical center, we took a systematic look at our annual data on academic promotions by percentage rank for faculty who are women, Asian and underrepresented in medicine. In this manuscript, we report our original data for the time period of 2001 to 2019 for the percentage of faculty who were underrepresented by rank for the years 2001, 2008, 2014 and 2019. As displayed in Fig. 2, our data show that while we have increased the number of underrepresented faculty, we have not had a significant increase 
Fig. 2 Graph shows percentage of Boston Children's Hospital faculty who were underrepresented in medicine by academic rank from 2001 to 2019. URiM underrepresented in medicine

\section{$30 \%$}

$25 \%$

$20 \%$

$15 \%$

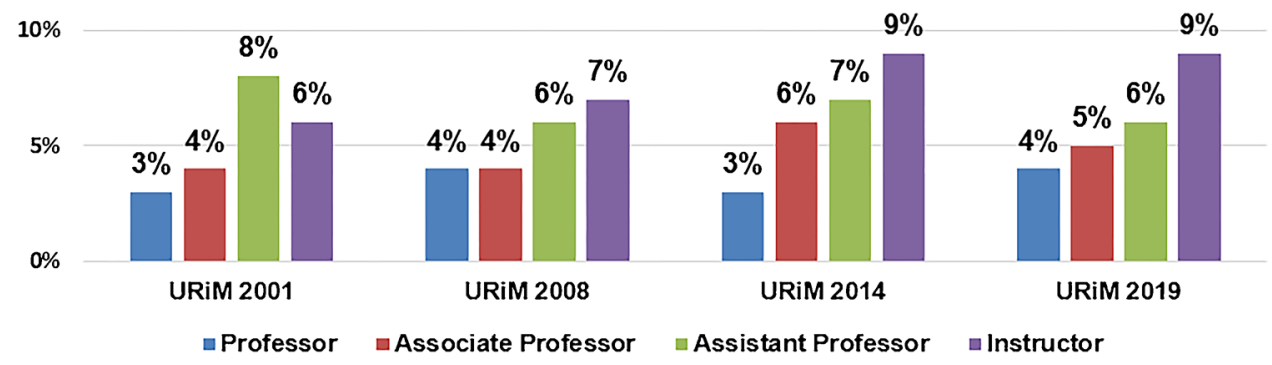

in the percentage of underrepresented faculty at each rank over this time period, and that our medical center needs to direct resources and expertise toward initiatives focused on increasing the academic advancement of our underrepresented faculty. Similarly, at Harvard Medical School there has been an increase in diverse faculty over the 1980-2020 time period, yet the percentage of underrepresented-in-medicine faculty (defined here as Black, Hispanic and Native American) has not changed, and these data are shown in Fig. 3 [21].

Fig. 3 Graph shows Harvard Medical School underrepresented-in-medicine and Asian or Pacific Islander full-time faculty (1980-2020) [21]. URM underrepresented in medicine; in this figure, underrepresented in medicine refers to Black, Hispanic and Native American faculty
In 2021, we launched a pilot program at Boston Children's Hospital for early career faculty who were underrepresented in medicine, an intentional step to address this opportunity gap. This innovative pilot program is a 2-year collaboration among the OHEI, the Department of Education and the Office of Faculty Development. Integral to this program is its co-directorship and sponsorship by hospital senior executive leaders who are committed to increasing the numbers and the academic advancement of underrepresented faculty. The early career faculty participate in

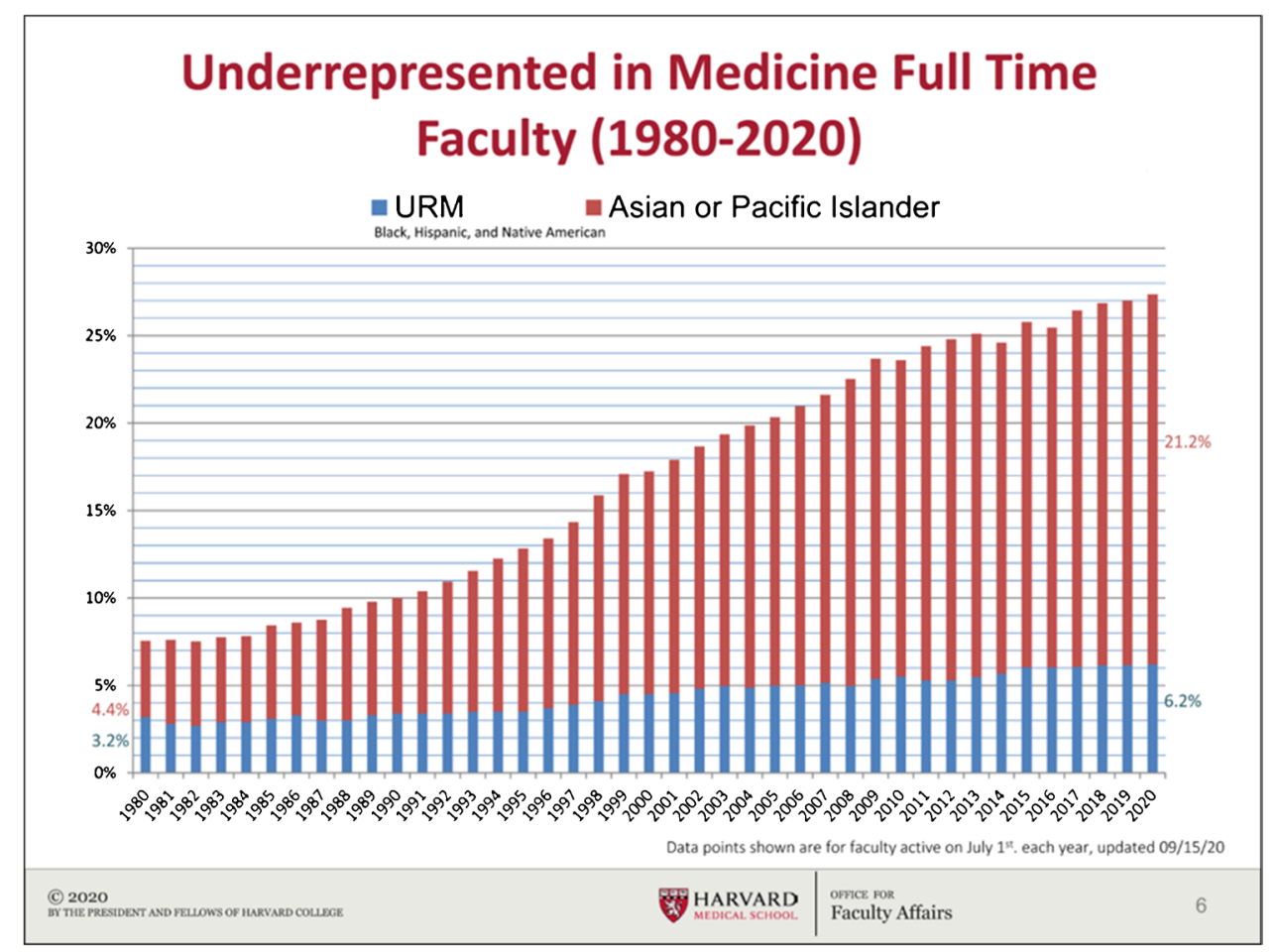


monthly career advancement seminars that are focused on leadership development and academic scholarship/productivity and led by experts in medical and surgical education, legal/general counsel, and research and faculty development. Monthly, the early career underrepresented faculty meet 1:1 with senior faculty and also attend leadership development courses outside of Boston Children's so they can build a larger framework for their career development.

In keeping with this focus on diverse faculty, the Office of Faculty Development reviewed the demographic data of faculty by gender and race/ethnicity and highlighted the findings to department chairs and senior leadership related to the lack of significant progress in recruitment and promotion of underrepresented faculty, similar to the trends reported by other Harvard Medical School-affiliated hospitals and academic centers nationally [21]. These data created additional urgency for the establishment of the OHEI to systematically advance programs to address the diversity of faculty, trainees and staff.

When asked in the 2017 Office of Faculty Development Survey (unpublished data), our faculty wanted the OHEI to focus on addressing recruitment and retention of diverse faculty (66\%) and fellows (49\%), and training on unconscious bias (51\%). Faculty commented that gender disparities require attention, as does inclusion of people with disabilities. In the 2019 Office of Faculty Development Survey (unpublished data), faculty reported that the four most important issues were recruiting and retaining diverse faculty $(71 \%)$; recruiting and retaining diverse trainees (55\%); promoting culturally effective pediatric care, including training in unconscious bias (51\%); and providing career networking opportunities (46\%). Additional recommendations from respondents included requiring effective training in EDI, EDI metrics to hold divisions/departments/programs accountable, addressing inequities in faculty promotion and salary, and establishing clear processes for faculty/ staff to report practices that they felt were disrespectful or discriminatory.

We also undertook a systematic analysis of the results of the dedicated funding of research grants for underrepresented faculty as it relates to academic promotion and retention. Our Faculty Career Development Fellowships provided transitional grants to sustain the research productivity in basic science and clinical/translational research and career growth of junior faculty. The faculty fellowships are $\$ 50,000 /$ year for 2 years in direct costs; a portion of the awards are designated for faculty who are underrepresented in medicine or science or who have family responsibilities such as being primary caregivers for children or parents. Recipients reported that the fellowships increased their contact with mentors and department chairs across the medical center and Harvard Medical School, enhanced their career trajectory and promotions, strengthened their academic productivity and their "ability to take risks in science," helped in building collaborations, provided pilot data for grants, and supported work-life balance (unpublished Office of Faculty Development annual report).

The results of our analysis of the underrepresented faculty recipients of the career development fellowships are as follows: Of the 167 Faculty Career Development recipients from 2012 to $2019,8.4 \%$ (14/167) were underrepresented faculty. While women made up $64.7 \%$ of all recipients, only $57.1 \%(8 / 14)$ of underrepresented faculty candidates were women. This difference was not statistically significant $(P=0.38)$. Additional funding was received by $71.4 \%(10 / 14)$ of underrepresented faculty recipients and $77.8 \%(119 / 153)$ of non-underrepresented faculty recipients $(P=0.59)$. While the overall retention rate was $73.1 \%$, underrepresented faculty retention rates were lower than non-underrepresented faculty rates $(57.1 \%$ vs. $74.5 \%)$. This difference, however, was not statistically significant $(P=0.16)$. Of those who stayed at Boston Children's Hospital, 87.5\% (7/8) of underrepresented faculty recipients were promoted compared to $68.4 \%$ (78/114) of non-underrepresented faculty recipients (not statistically significant, $P=0.19$ ). These data provide the justification for intentionality in our underrepresented faculty development and retention efforts.

\section{Discussion}

We reviewed our pediatric academic medical center's creation of the OHEI and learned four key lessons. The four lessons were to (1) prioritize EDI initiatives as strategic goals endorsed by the board and senior executive leadership; (2) collaborate with multidisciplinary departments, programs, offices and experts; (3) take an academic approach and create educational and research EDI initiatives; and (4) commit to intentionality and accountability for all EDI initiatives (Fig. 4). It is important to underscore that we operationalized these EDI initiatives, with multi-specialty and interprofessional collaboration among pediatric subspecialists (pediatric radiologists), pediatricians, nurses, researchers and senior executive hospital leaders. Intentional efforts to build EDI initiatives must be implemented within the overall institutional priorities of a medical center and include: developing equitydriven educational programming and research initiatives; creating training opportunities for health equity research; improving collection and analysis of pediatric patient race, ethnicity and language demographic data and health equity-quality metrics to identify and address health care disparities [11,22]; creating hospital-wide education to address unconscious biases, create inclusive learning and working environments in health care settings [23] and build pipeline programs at high school and college levels; 


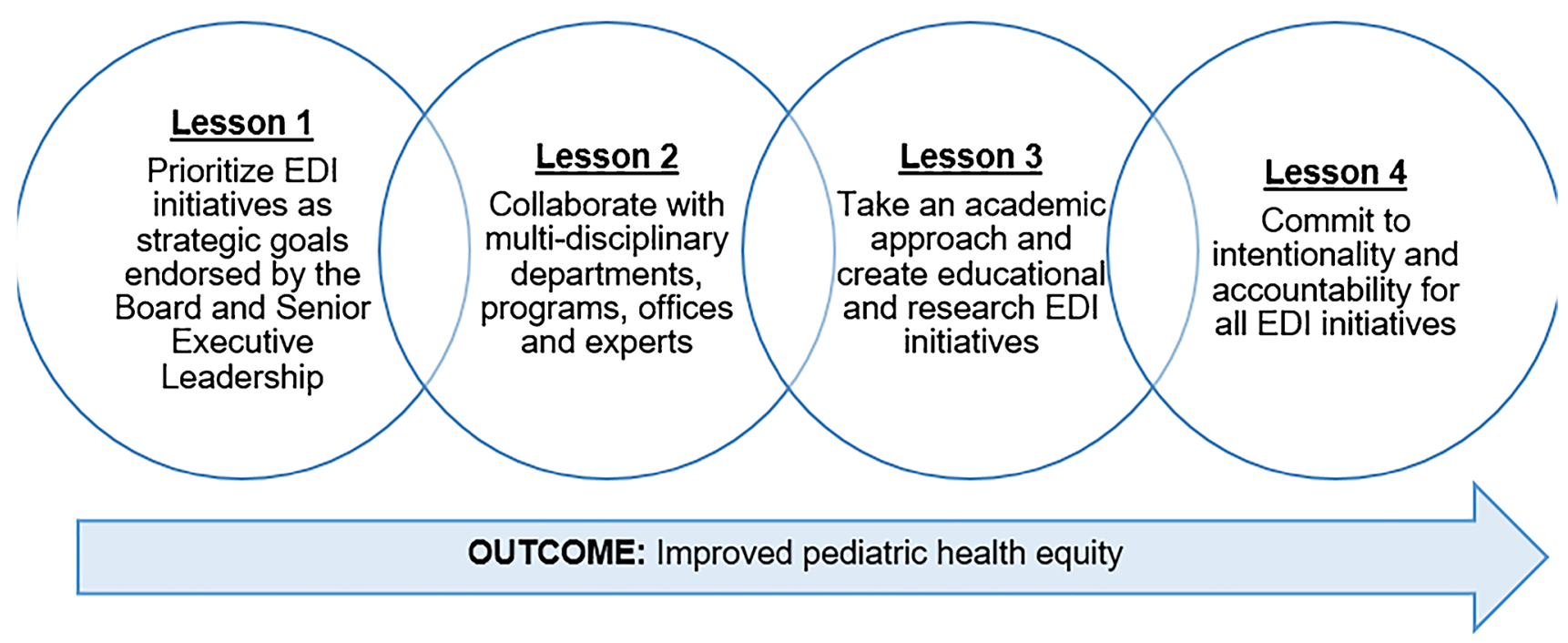

Fig. 4 Lessons learned in creating a Health Equity and Inclusion Office in an academic pediatric medical center. EDI equity, diversity and inclusion

and expanding faculty development and research grant programs to increase underrepresented faculty workforce diversity, retention and academic advancement/promotion [2]. The original data presented here provide the evidence for the priorities addressed and highlight the lessons learned in the creation of an office of health equity and inclusion within an academic medical center with the overall aim to be intentional in the important work to improve pediatric health equity.

In summary, the creation of the Office of Health Equity and Inclusion at our pediatric academic medical center, the strategies selected, priorities addressed and lessons learned were all based on the intentional engagement of and collaboration with key clinical, administrative and senior executive leaders across Boston Children's Hospital. We are grateful for opportunities to engage with other pediatric academic centers to share our challenges and innovations as we address health equity and inclusion for our staff, trainees and faculty as well as the patients and families and communities we serve.

\section{Conclusion}

Boston Children's Hospital chose to establish an Office of Health Equity and Inclusion that is an enterprise-level office reporting directly to senior executive hospital leadership. In this manuscript, we present actionable and intentional steps taken in our pediatric academic medical center's equity, diversity and inclusion strategy to engage all sectors of its workforce and to implement institutional priorities and corporate goals focused on improving pediatric health equity.
Acknowledgments Alan M. Leichtner, MD, MSHPEd, S. Jean Emans, MD, and Kevin B. Churchwell, MD, contributed equally. The authors wish to acknowledge the significant contributions of Eileen Sporing, MSN, RN, FAAN, former senior vice president of Patient Care Services and former chief nursing officer, Boston Children's Hospital, in the creation of the Diversity and Cultural Competency Council. The authors also wish to acknowledge Elliott Kellman, executive vice president, system chief human resources officer, for his collaboration in the work of the Boston Children's Declaration on Equity, Diversity and Inclusivity; and Judy Zola, MBA, director of Learning and Development, who is the Human Resources co-chair of the Equity, Diversity and Inclusion Council (formerly called the Diversity and Cultural Competency Council).

\section{Declarations}

Conflicts of interest Valerie L. Ward, MD, MPH, is the principal investigator on a Boston Children's Hospital Academy for Teaching and Educational Innovation and Scholarship Healthcare Professional Education Grant (2020-2021), which funded this study. The authors declare no conflicts of interest.

\section{References}

1. Braveman P, Arkin E, Orleans T et al (2017) What is health equity? And what difference does a definition make? Robert Wood Johnson Foundation, Princeton

2. Laurencin CT, National Academies of Sciences, Engineering, and Medicine (2020) The impacts of racism and bias on Black people pursuing careers in science, engineering, and medicine: proceedings of a workshop. The National Academies Press, Washington, DC

3. Bucknor MD, Villanueva-Meyer JE, Kumar V et al (2019) Diversity and inclusion efforts in University of California, San Francisco radiology: reflections on 3 years of pipeline, selection, and education initiatives. J Am Coll Radiol 16:1716-1719 
4. Crowley AL, Damp J, Sulistio MS et al (2020) Perceptions on diversity in cardiology: a survey of cardiology fellowship training program directors. J Am Heart Assoc 9:e017196

5. DeBenedectis CM, Heitkamp DE, England E et al (2020) A program director's guide to cultivating diversity and inclusion in radiology residency recruitment. Acad Radiol 27:864-867

6. Mohile NA, Spector AR, Ebong IM et al (2021) Developing the neurology diversity officer: a roadmap for academic neurology departments. Neurology. https://doi.org/10.1212/WNL.00000 00000011460

7. Ellis J, Otugo O, Landry A, Landry A (2020) Interviewed while Black. N Engl J Med 383:2401-2404

8. Emans SJ, Goldberg CT, Milstein ME, Dobriner J (2008) Creating a faculty development office in an academic pediatric hospital: challenges and successes. Pediatrics 121:390-401

9. Lee LK, Mulvaney-Day N, Berger AM et al (2016) The patient passport program: an intervention to improve patient-provider communication for hospitalized minority children and their families. Acad Pediatr 16:460-467

10. Samuels RC, Ward VL, Melvin P et al (2015) Missed appointments: factors contributing to high no-show rates in an urban pediatrics primary care clinic. Clin Pediatr 54:976-982

11. Cowden JD, Flores G, Chow T et al (2020) Variability in collection and use of race/ethnicity and language data in 93 pediatric hospitals. J Racial Ethn Heal Disparities 7:928-936

12. The Disparities Solutions Center (2020) Pediatric Health Equity Collaborative. Web site. https://www.mghdisparitiessolutions.org/ phec. Accessed 17 Feb 2020

13. Tan-McGrory A, Bennett-AbuAyyash C, Gee S et al (2018) A patient and family data domain collection framework for identifying disparities in pediatrics: results from the pediatric health equity collaborative. BMC Pediatr 18:1-13
14. Gooding HC, McCarty C, Millson R et al (2016) The Boston Children's Hospital Academy: development and initial assessment of a hospital-based teaching academy. Acad Med 91:1651-1654

15. Pletcher BA, Rimsza ME, Basco WT et al (2013) Enhancing pediatric workforce diversity and providing culturally effective pediatric care: implications for practice, education, and policy making. Pediatrics 132:e1105-1116

16. Toomey SL, Chien AT, Elliott MN et al (2013) Disparities in unmet need for care coordination: the National Survey of Children's Health. Pediatrics 131:217-224

17. Guevara JP, Adanga E, Avakame E, Carthon MB (2013) Minority faculty development programs and underrepresented minority faculty representation at US medical schools. JAMA 310:2297-2304

18. Reede JY (2014) Minority faculty development programs at US medical schools. JAMA 311:1157-1158

19. Trent M, Dooley DG, Dougé J et al (2019) The impact of racism on child and adolescent health. Pediatrics 144:1-14

20. Evans MK, Rosenbaum L, Malina D et al (2020) Diagnosing and treating systemic racism. N Engl J Med 383:274-276

21. Harvard Medical School Office for Faculty Affairs (2020) Faculty demographics. https://fa.hms.harvard.edu/faculty-demographics. Accessed 10 Feb 2021

22. Chin MH (2020) Advancing health equity in patient safety: a reckoning, challenge and opportunity. BMJ Qual Saf. https://doi.org/ 10.1136/bmjqs-2020-012599

23. Brottman MR, Char DM, Hattori RA et al (2020) Toward cultural competency in health care: a scoping review of the diversity and inclusion education literature. Acad Med 95:803-813

Publisher's note Springer Nature remains neutral with regard to jurisdictional claims in published maps and institutional affiliations.

\section{Authors and Affiliations}

\section{Valerie L. Ward ${ }^{1,2,3,4} \cdot$ Nicole W. Tennermann ${ }^{2} \cdot$ Geeranan Chuersanga ${ }^{2} \cdot$ Patrice Melvin $^{2,3,5} \cdot$ Maxine E. Milstein $^{6}$. Jonathan A. Finkelstein ${ }^{4,7,8}$. Michele M. Garvin ${ }^{9}$. Laura J. Wood ${ }^{10}$ - Nina A. Rauscher ${ }^{11}$. Peter C. Laussen ${ }^{4,11,12}$. Alan M. Leichtner ${ }^{4,8,13,14} \cdot$ S. Jean Emans $s^{4,6,8,15} \cdot$ Kevin B. Churchwell ${ }^{4,16}$}

1 Department of Radiology, Boston Children's Hospital, 300 Longwood Ave., Boston, MA 02115, USA

2 Office of Health Equity and Inclusion, Boston Children's Hospital, Boston, MA, USA

3 Sandra L. Fenwick Institute for Pediatric Health Equity and Inclusion, Boston Children's Hospital, Boston, MA, USA

4 Harvard Medical School, Boston, MA, USA

5 Center for Applied Pediatric Quality Analytics, Boston Children's Hospital, Boston, MA, USA

6 Office of Faculty Development, Boston Children's Hospital, Boston, MA, USA

7 Program for Patient Safety and Quality, Boston Children's Hospital, Boston, MA, USA

8 Department of Pediatrics, Boston Children's Hospital, Boston, MA, USA

9 Office of General Counsel, Boston Children's Hospital, Boston, MA, USA
10 Nursing and Patient Care Operations, Boston Children's Hospital, Boston, MA, USA

11 Department of Health Affairs, Boston Children's Hospital, Boston, MA, USA

12 Department of Cardiology, Boston Children's Hospital, Boston, MA, USA

13 Division of Gastroenterology, Boston Children's Hospital, Boston, MA, USA

14 Department of Education, Boston Children's Hospital, Boston, MA, USA

15 Division of Adolescent/Young Adult Medicine, Boston Children's Hospital, Boston, MA, USA

16 Office of the CEO and President, Boston Children's Hospital, Boston, MA, USA 